\title{
Optimization of neutrino fluxes for future long baseline neutrino oscillation experiment
}

\section{Silvestro Di Luise*}

ETH Zurich Institute for Particle Physics

E-mail: silvestro.di.luise@cern.ch

\section{A. Longhin}

IRFU CEA-Saclay,

E-mail: andrea.longhin@cea.fr

\section{A. Rubbia}

ETH Zurich Institute for Particle Physics,

E-mail: andre.rubbia@cern.ch

\begin{abstract}
We present an optimization of the neutrino beam which could be produced at CERN and aimed to the newly proposed deep-underground neutrino observatory LAGUNA-LBNO located at distance of about $2300 \mathrm{~km}$ (Phyäsalmi mine, Finland). Specific scenarios for the proton driver and the far detectors have been investigated. In particular, the flux predictions have been obtained by means of a full GEANT4 simulation of the primary proton beam interaction in the target, the secondary hadrons focusing system (e.g. horn-reflector layout) and the decay pipe. A dedicated algorithm has been then developed to search for the optimal configuration of the beam line by scanning the multidimensional space of the desgin parameters. The aim is to maximize the LAGUNA-LBNO physics performance in terms of the discovery potential for both the $\mathrm{CP}$ violation (CPV) phase $\delta_{C P}$ and the mass hierarchy $(\mathrm{MH})$. First results are presented in this proceedings.
\end{abstract}

36th International Conference on High Energy Physics

4-11 July 2012

Melbourne, Australia

\footnotetext{
${ }^{*}$ Speaker.
} 

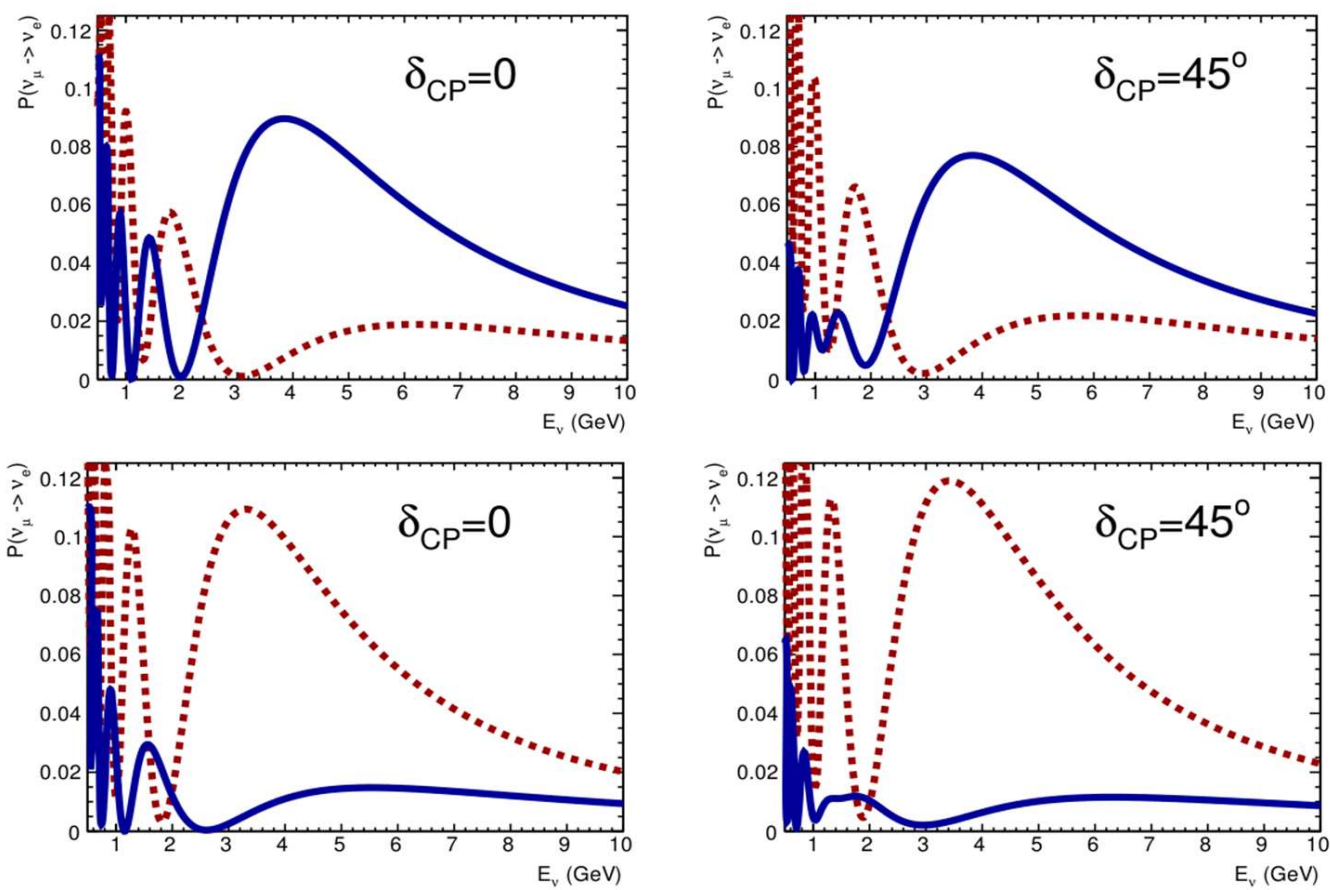

Figure 1: Oscillation probability of $v_{\mu} \rightarrow v_{e}$ (solid line) and $\bar{v}_{\mu} \rightarrow \bar{v}_{e}$ for two values of $\delta_{C P}$. Top: normal hierarchy (NH, $\left.\Delta m_{31}^{2}>0\right)$. Bottom:inverted hierarchy ( $\left.\mathrm{IH}, \Delta m_{31}^{2}<0\right)$. The value of $\sin ^{2} 2 \theta_{13}$ is set to 0.09 .

The LAGUNA-LBNO collaboration has submitted and Expression of Interest (EoI)[1] for a next generation neutrino oscillation experiment between CERN and the Phyäsalmi mine in Finland (depth: 400 m.w.e.), $2300 \mathrm{~km}$ distant. The main goals of the LBNO physics program are the determinatin of the Mass Hierachy $(\mathrm{MH})$ and the measurement of the CP phase, $\delta_{C P}$, in the leptonic sector. The proposed experimental setup consists of a new conventional neutrino beam facility coupled with a next generation underground neutrino observatory composed of a double phase liquid Argon detector and a magnetized iron calorimeter. Other physics goals achievable with a deep undergroung massive neutrino detector are the search for proton decay and neutrino astrophysics searches.

In Fig. 1 is shown the energy dependence of the oscillation probability, for both $v_{\mu} \rightarrow v_{e}$ and $\bar{v}_{\mu} \rightarrow \bar{v}_{e}$ transitions as expected for a baseline of $2300 \mathrm{~km}$. Two values of $\delta_{C P}$ (i.e.: 0 and $\pi / 4$ ) and both mass ordering hypothesis are considered. The distributions illustrate qualitatively that the spectral information provides an unambiguous determination of the oscillations parameters and allows discriminating between the two CP-conserving scenarios. The $\delta_{C P}$-phase and matter effects introduce a well-defined energy dependence of the oscillation probability. As a consequence, the neutrino energy spectrum of the oscillated events need to be experimentally reconstructed with sufficiently good resolution in order to distinguish first and second maximum and extract unambiguous information on the oscillation parameters. Moreover a wide-band beams is needed in order to cover the several maxima. The neutrino beamline design is therefore a central component of the LBNO optimization since it will directly impact the long baseline physics reach, affecting for instance the mass hierarchy sensitivity, the $\mathrm{CP}$ violation reach and the study of the other oscillation channels like the tau appearance. 


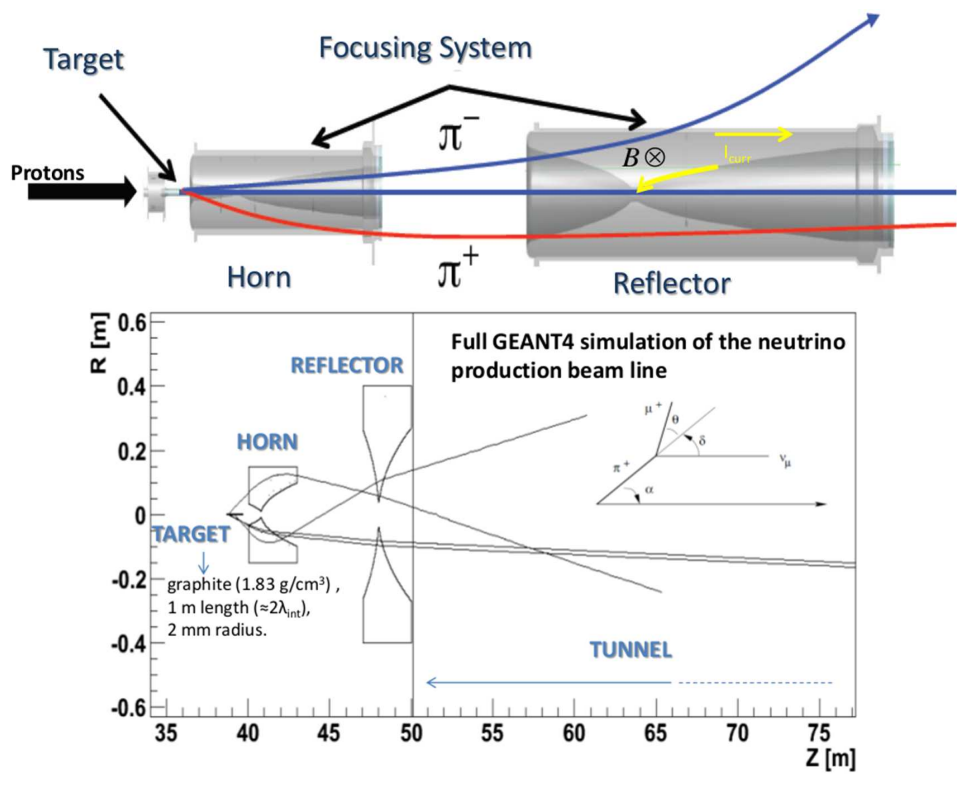

Figure 2: Top: Schematic drawing of a focusing system. Bottom: Example of a GEANT4 event with hadrons produced in the target, focused through the horns and transported into the decay tunnel.

Conventional neutrino beams from high-energy proton accelerators have been instrumental discovery tools in particle physics. Neutrino beams are derived from the decays of charged $\pi$ and $\mathrm{K}$ mesons, which in turn are created from proton beams striking thick nuclear targets. The precise selection and manipulation of the $\pi / \mathrm{K}$ beam controls both the energy spectrum and the flavour composition of the neutrino beam. In Fig. 2 is shown the conceptual design of a charged particle focusing system which cosists of one or more "horn". Each horn consits of two coaxial conductors which encompass a closed volume. At each beam spill a pulsed current magnetizes the horn generating a toroidal magnetic field (i.e.: varying as $1 / \mathrm{r}$ if the $\mathrm{r}$ is the distance from the axis) of few Tesla in the horn volume. Such a sytem is used to focus charged particles ( $\pi$ and $\mathrm{K}$ ) produced in the interaction of the primary proton beam in the target in the direction of the neutrino far detector. One can choose to focus neutrinos or antineutrinos by changing the horn current polarity.

In the initial phase of LBNO, it is assumed that the neutrino beam will be produced from high-energy protons fast extracted from the CERN SPS at an energy of $400 \mathrm{GeV}$ with a repetition cycle time of 6 seconds. In order to compute the neutrino fluxes, a detailed geometry has been implemented in a GEANT4[2]-based program and full simulation with tracking of all secondary particles has been performed. The neutrino beam line is tentatively composed of a target, two horns and a decay volume. The target is modeled as a $1 \mathrm{~m}$ long cylinder of graphite with density $\rho=$ $1.85 \mathrm{~g} / \mathrm{cm}^{3}$ and $2 \mathrm{~mm}$ radius. The focusing system is based on a pair of parabolic horns denoted as horn (upstream) and reflector (downstream) according to the current terminology. An example of a simulated event is shown in Fig. 2.

A first attempt to parametrically optimize the focusing system has been performed to maximize a specific physics sensitivity. The optimization relies on a parametric model of the horn and reflector profiles. In addition to the parameters related to the shape of the horn and the reflector, additional degrees of freedom are: the distance between the horn and reflector, the length and ra- 

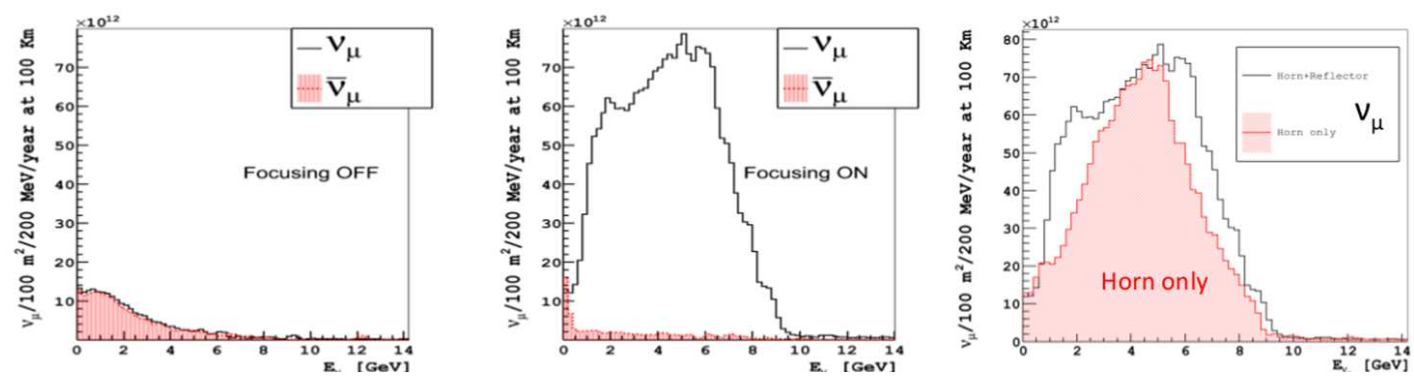

Figure 3: Right and Central: Neutrinos and anitineutrinos flux with and without focusing. Left: Neutrino flux when the reflector is not magnetized (shaded area) compared to the flux obtained focusing the hadrons with both the horn and the reflector.
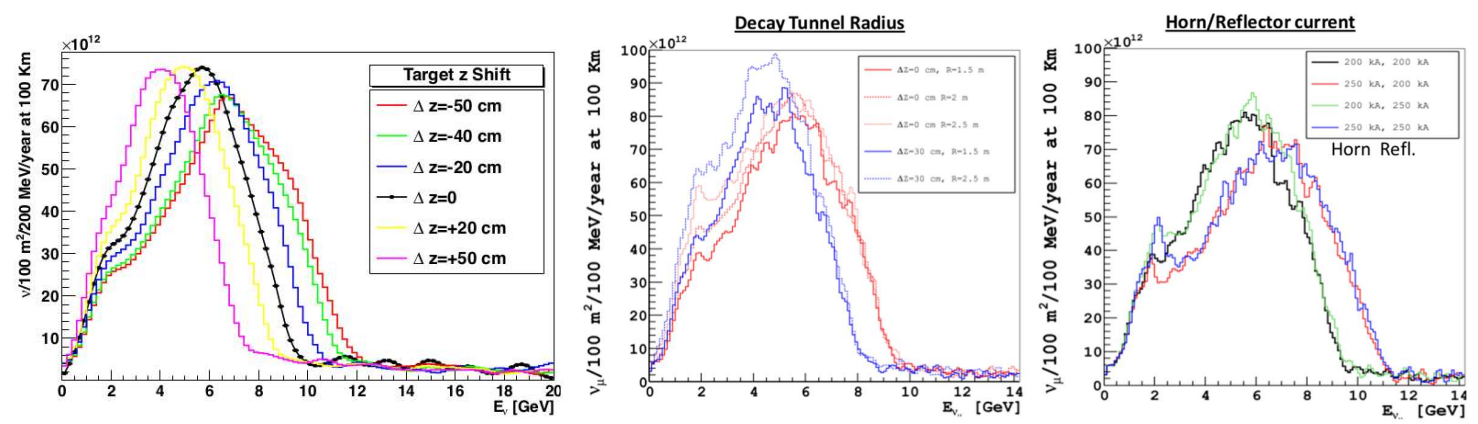

Figure 4: Neutrino fluxes for different choices of the target-horn distance, decay tunnel radius and horn/reflector current.

dius of the decay tunnel, the longitudinal position of the target and the currents circulating in the horns. The criterium used to optimize the neutrino flux energy distribution is the maximization of the sensitivity to the CPV. The (anti)neutrino reconstructed energy and the transverse missing momentum $p_{T}^{\text {miss }}$ distribution have been used in the oscillation analysis. The latter information was used in order to reduce the background induced by $v_{\tau}$ interactions: $v_{\mu} \rightarrow v_{\tau} \rightarrow \tau \rightarrow e v_{e} v_{\tau}$.

The systemtic error on the mormalization of both the signal and the background from the beam electron contamination is assumed to be $\pm 5 \%$ The $\tau$ normalization which is poorly known due to the large cross section uncertainties is set to $\pm 50 \%$. Both the $v$ NC and $v_{\mu} \mathrm{CC}$ backgrounds have a systematic error of $\pm 10 \%$, while the relative normalization of positive and negative horn polarity has an uncertainty of $\pm 5 \%$.

The considered target fiducial mass is $20 \mathrm{kton}$.

For comparison, all the (anti)neutrino fluxes are normalized to a distance of $100 \mathrm{~km}$ and a target surface of $10 \times 10 \mathrm{~m}^{2}$.

In Fig. 3 is visibile how the focusing of positive particles affects the neutrino and antineutrino flux. It is clear also the crucial role of the reflector in the focusing of low momentum hadrons which produced neutrinos in the energy range of the second oscillation maximum.

The effect of the different configuration of the focusing layout on the neutrino flux energy distribution is shown in Fig. 4. In particular should be stressed how by reducing the distance between the target and the horn a shift towards low energy is obtained in the neutrino flux. This is due to an increase of the acceptance for low energy hadrons which are emitted at a higher angle 

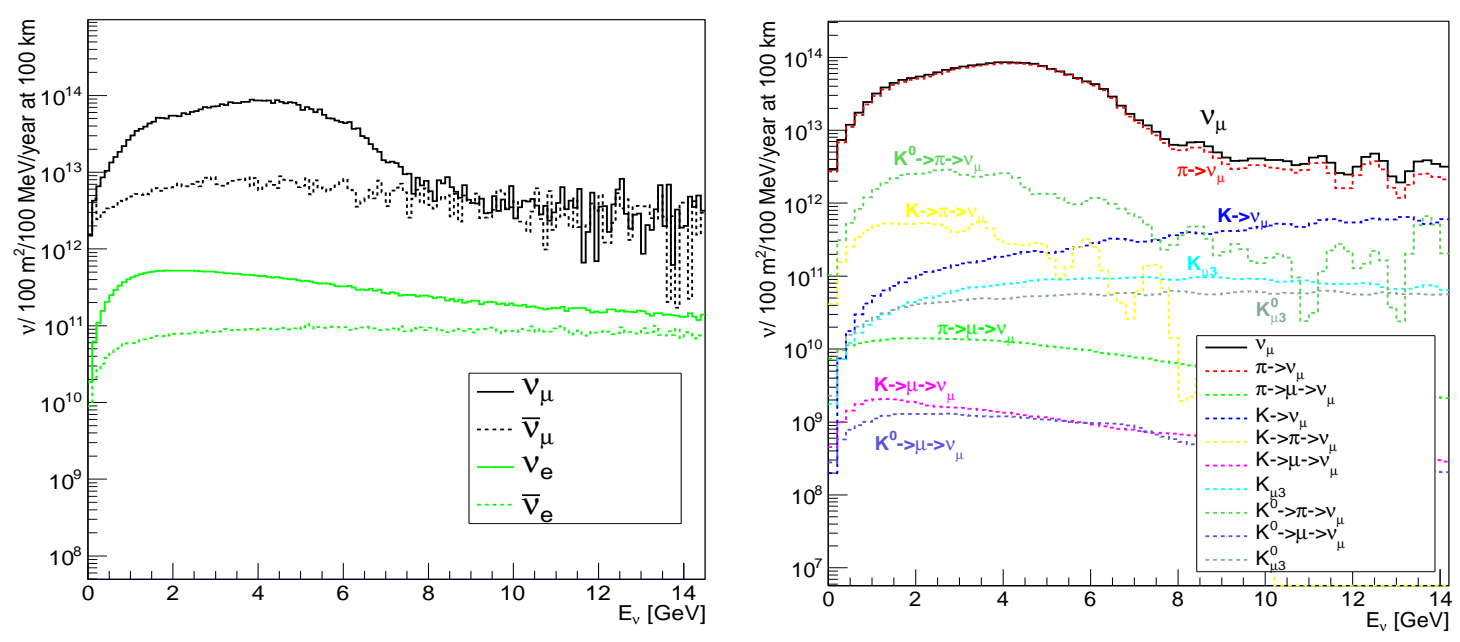

Figure 5: Detailed flux composition for the improved beam cofiguration.
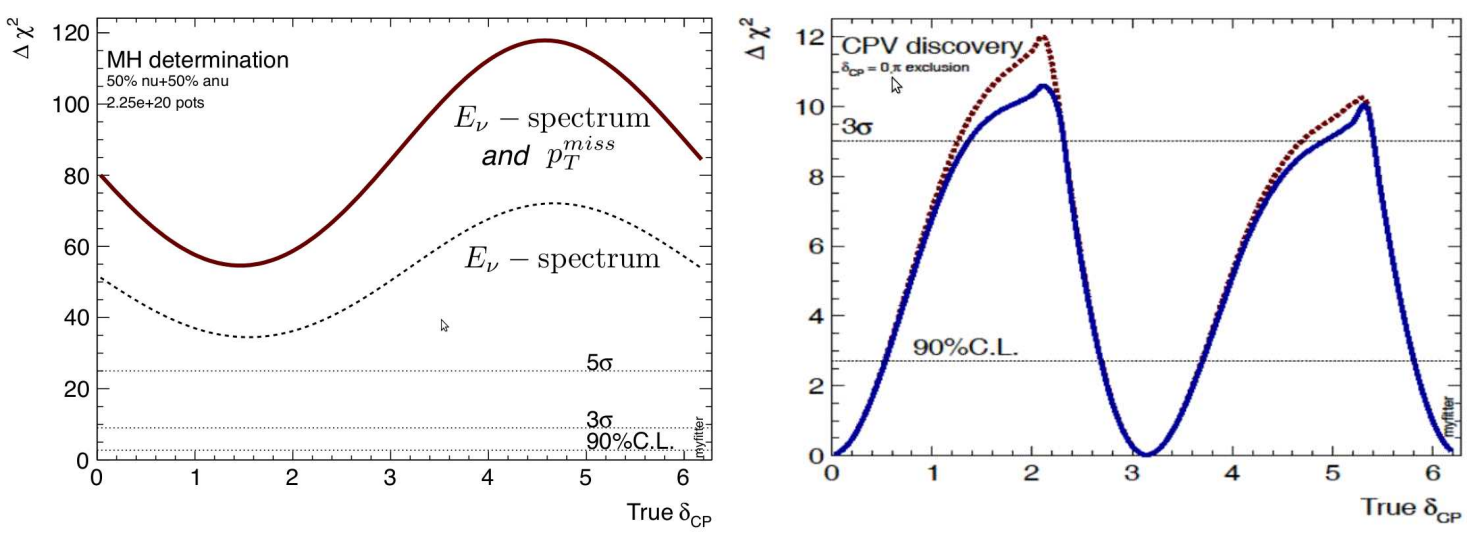

Figure 6: Left: $\Delta \chi^{2}$ of the mass hierarchy discriminant as a function of true $\delta_{C P}\left(2.25 \times 10^{20}\right.$ p.o.t., $50 \%: 50 \%$ neutrino:antineutrino beam sharing). Right: $\Delta \chi^{2}$ of the CPV discriminant as a function of true $\delta_{C P}\left(1.5 \times 10^{21}\right.$ p.o.t., $25 \%: 75 \%$ neutrino:antineutrino beam sharing). The blue curve corresponds to the case with all systematic errors included. The dashed brown curve is the case where all energy correlated errors are set to zero and the average Earth density error is reduced for $5 \%$ to $1 \%$.

with respect to the horn axis.

From a preliminary study of the optimization a beamline layout where the horn-reflector distance is of 2 meters, the current is $200 \mathrm{kA}$ and the tunnel length and radius are $400 \mathrm{~m}$ and $2 \mathrm{~m}$ respectively is considered. The target is placed inside the first horn for the $50 \%$ of the length. Detailed information on the chosen horn shape parametrization can be found in[1]. The corresponding neutrino flux is shown in Fig. 5.

Fig. 6 shows the results obtained with the improved flux in terms of sensitivity to the Mass Hierarchy and the CPV. The obtained significance on the Mass Hierarchy determination is above $5 \sigma$ over the entire range of $\delta_{C P}$ values. The CPV discovery potential is presented in Fig. 6 as a function of the $\delta_{C P}$. It exhibits the expected double peak structure with two zero values at $\delta_{C P}=0$ and $\pi$, stemming from the definition of the $\chi^{2}$, which defines the CPV discovery as the exclusion 
of 0 and $\pi$. With $1.5 \times 10^{21}$ p.o.t., the existence of CP-violation (CPV) can be demonstrated at the $90 \%$ C.L. for $\approx 70 \%$ of the $\delta_{C P}$ parameter space.

\section{References}

[1] A. Stahl et al., CERN-SPSC-2012-021 (SPSC-EOI-007).

[2] S. Agostinelli et al., Nuclear Instruments and Methods in Physics Research Section A: Accelerators, Spectrometers, Detectors and Associated Equipment 506, 250 (2003). 